\title{
Neutrophil-to-lymphocyte ratio, platelet-to-Iymphocyte ratio, and their dynamic changes during chemotherapy is useful to predict a more accurate prognosis of advanced biliary tract cancer
}

\author{
Kyoung-Min Cho ${ }^{1,3}$, Hyunkyung Park ${ }^{1}$, Do-Youn Oh ${ }^{1,2}$, Tae-Yong Kim ${ }^{1}$, Kyung-Hun \\ Lee $^{1,2}$, Sae-Won Han ${ }^{1,2}$, Seock-Ah Im ${ }^{1,2}$, Tae-You Kim ${ }^{1,2}$ and Yung-Jue Bang ${ }^{1,2}$ \\ ${ }^{1}$ Department of Internal Medicine, Seoul National University Hospital, Seoul National University College of Medicine, Seoul, \\ Republic of Korea \\ ${ }^{2}$ Cancer Research Institute, Seoul National University College of Medicine, Seoul, Republic of Korea \\ ${ }^{3}$ Department of Internal Medicine, School of Medicine, Kyung Hee University, Seoul, Republic of Korea \\ Correspondence to: Do-Youn Oh, email: ohdoyoun@snu.ac.kr
}

Keywords: inflammation, prognosis, advanced biliary tract cancer, neutrophil-to-lymphocyte ratio

Received: September 06, 2016 Accepted: November 24, $2016 \quad$ Published: November 30, 2016

\section{ABSTRACT}

Background and Purpose: Systemic inflammation is known to promote carcinogenesis in biliary tract cancer (BTC). Neutrophil-to-lymphocyte ratio (NLR) and platelet-to-lymphocyte ratio (PLR) are indicative of systemic inflammation. We evaluated the clinical significance of systemic inflammation measured by NLR and PLR in patients with advanced BTC. Additionally, we also co-analyzed the dynamics of NLR and PLR during chemotherapy.

Methods: We reviewed 450 patients with unresectable BTC receiving palliative chemotherapy. NLR and PLR were obtained before initiation of palliative chemotherapy. Changes in NLR, PLR were obtained by subtracting the initial value from the value obtained after progression of chemotherapy.

Results: Higher systemic inflammation status also had relation with a primary tumor site $(p=0.003)$ and higher levels of CEA $(p=0.038)$. The ROC cut-off values of NLR and PLR for predicting overall survival (OS) were 3.8 and 121, respectively. Patients with a high NLR or PLR had worse OS independently in multivariate analysis (6.90 vs. 9.80 months, $p=0.002 ; 7.83$ vs. 9.90 months, $p=0.041$, respectively). High NLR with increased NLR after chemotherapy is associated with worse OS and progression-free survival (PFS) $(p<0.001, p=0.013$ respectively). Results are similar for PLR.

Conclusion: Systemic inflammation represented by NLR and PLR, predicts the OS of patients with advanced BTC who are receiving palliative chemotherapy. In addition, considering NLR/PLR with a dynamic change of NLR/PLR during chemotherapy might help to predict a more accurate prognosis.

\section{INTRODUCTION}

Biliary tract cancers (BTCs) are tumors that are rare and have a poor prognosis. In Western countries, BTCs comprise less than $2 \%$ of all malignancies [1], but in Korea, they are more common, accounting for approximately $4 \%$ of malignancies [2]. Most patients who undergo surgical resection for BTC, generally experience a recurrence after a short disease-free period [3]. Overall survival (OS) in patients with advanced or metastatic BTC is less than 1 year [4]. The known prognostic factors include albumin, Eastern Cooperative Oncology Group (ECOG) Performance Status (PS), and serum carcinoembryonic antigen (CEA) level [5]; however, there is a lack of prognostic factors that can accurately predict the prognosis of BTC.

Chronic inflammation is known to promote carcinogenesis [6], which is triggered by cytokine or 
Table1: Baseline Characteristics of Patients.

\begin{tabular}{|c|c|}
\hline Characteristics & Patients $(n=450)$ \\
\hline \multicolumn{2}{|l|}{ Age - yr } \\
\hline Median & 61 \\
\hline Range & $26-84$ \\
\hline \multicolumn{2}{|l|}{ Sex-no $(\%)$} \\
\hline Male & $295(65.6 \%)$ \\
\hline Female & $155(34.4 \%)$ \\
\hline \multicolumn{2}{|l|}{ Primary tumor site - no. $(\%)$} \\
\hline $\mathrm{ICC}$ & $214(47.6 \%)$ \\
\hline $\mathrm{GB} \mathrm{Ca}$ & $151(33.6 \%)$ \\
\hline Extrahepatic BTC & $54(12.0 \%)$ \\
\hline $\mathrm{AoV} \mathrm{Ca}$ & $31(6.9 \%)$ \\
\hline \multicolumn{2}{|l|}{ ECOG PS- no. $(\%)$} \\
\hline $0-1$ & $401(89.1 \%)$ \\
\hline$\geq 2$ & $49(10.9 \%)$ \\
\hline CEA (ng/ml) median (range) & $2.8(0.5-5410.0)$ \\
\hline CA $19-9(\mathrm{U} / \mathrm{ml})$ median (range) & $134.9(1.0-2400000.0)$ \\
\hline Total bilirubin $(\mathrm{mg} / \mathrm{dl})$ median (range) & $0.9(0.1-11.7)$ \\
\hline Albumin (mg/dl) median (range) & $3.8(2.1-5.1)$ \\
\hline \multicolumn{2}{|l|}{$1^{\text {st }}$ chemotherapy - no } \\
\hline Gemcitabine -based & $204(45.3 \%)$ \\
\hline FU-based & $232(51.6 \%)$ \\
\hline Gemcitabine, FU -based & $14(3.1 \%)$ \\
\hline \multicolumn{2}{|l|}{ NLR } \\
\hline \multicolumn{2}{|l|}{ Median/ mean/ range } \\
\hline All BTC & $3.1 / 4.5 / 0.5-61.0$ \\
\hline ICC & $3.3 / 4.6 / 0.5-61.0$ \\
\hline $\mathrm{GB} \mathrm{Ca}$ & $3.1 / 4.3 / 0.6-49.3$ \\
\hline Extrahepatic BTC & $3.1 / 5.7 / 0.7-30$ \\
\hline $\mathrm{AoV} \mathrm{Ca}$ & $2.6 / 3.3 / 0.9-13.5$ \\
\hline \multicolumn{2}{|l|}{ PLR } \\
\hline \multicolumn{2}{|l|}{ Median, mean, range } \\
\hline All BTC & $155.8 / 200.4 / 48.6-4099.0$ \\
\hline ICC & $139.6 / 166.5 / 48.6-600.8$ \\
\hline $\mathrm{GB} \mathrm{Ca}$ & $166.4 / 218.3 / 58.8-2657.4$ \\
\hline Extrahepatic BTC & $184.3 / 295.0 / 57.9-4099.0$ \\
\hline $\mathrm{AoV} \mathrm{Ca}$ & $164.6 / 184.5 / 64.5-396.5$ \\
\hline \multicolumn{2}{|l|}{ Serum CRP (mg/dl) } \\
\hline Median, mean, range & $2.5 / 4.4 / 0.0-25.7$ \\
\hline \multicolumn{2}{|l|}{ All BTC } \\
\hline ICC & $2.4 / 4.4 / 0.0-24.9$ \\
\hline $\mathrm{GB} \mathrm{Ca}$ & $1.8 / 4.3 / 0.0-25.7$ \\
\hline Extrahepatic BTC & $3.2 / 4.2 / 0.0-20.1$ \\
\hline $\mathrm{AoV} \mathrm{Ca}$ & $4.0 / 5.4 / 0.2-16.3$ \\
\hline
\end{tabular}

Abbreviations: ICC, intrahepatic cholangiocarcinoma; BTC, biliary tract cancer; GB Ca, gallbladder cancer;AoV Ca, ampulla of Vater cancer; ECOG PS, Eastern Cooperative Oncology Group performance status;CEA, carcinoembryonic antigen; CA 19-9, cancer antigen 19-9; NLR, neutrophil-lymphocyte ratio; PLR, platelet-lymphocyte ratio; CRP, C-reactive protein

antitumor immunologic response within the tumor. Also, inflammation predisposes to cholangiocarcinoma [7]. A known risk factor for advanced BTC is chronic inflammation, which includes such things as gallstones, chronic hepatitis, primary sclerosing cholangitis, and liver fluke infection [8]. In gallbladder carcinoma, tumor necrosis factor- $\alpha$ (TNF- $\alpha$ ) promotes lymph node metastasis through nuclear factor- $\kappa \mathrm{B}-$ mediated [9]. In cholangiocarcinoma, the cytokine interleukin-6 (IL-6) is known to play a major role in tumor growth [10].

Although many biomarkers of systemic inflammation have recently been studied, excessive costs and technique factors prevent their clinical use. The most commonly investigated indicators of inflammation are those that are clinically most cost-effective and available, such as C-reactive protein (CRP) levels, platelet counts, and blood neutrophil counts [11]. Poor prognostic factors in various cancers include high neutrophil-to-lymphocyte ratio (NLR), high platelet-to-lymphocyte ratio (PLR), increased levels of neutrophils and platelets, and decreased levels of lymphocytes [12].

However, in BTC the role of NLR and PLR has 
Table 2: Comparison between the high NLR and low NLR groups.

\begin{tabular}{|c|c|c|c|}
\hline & $\begin{array}{l}\text { Low NLR group } \\
\text { NLR }<3.8(\mathrm{~N}=262)\end{array}$ & $\begin{array}{l}\text { High NLR group } \\
\text { NLR } \geq 3.8(\mathrm{~N}=173)\end{array}$ & $p$ value \\
\hline \multicolumn{3}{|l|}{ Age-yr } & 0.837 \\
\hline$\leq 65$ & $173(66.0 \%)$ & $112(64.7 \%)$ & \\
\hline$>65$ & $89(34.0 \%)$ & $61(35.3 \%)$ & \\
\hline \multicolumn{3}{|l|}{ Sex, no $(\%)$} & 0.010 \\
\hline Male & $160(61.1 \%)$ & $127(73.4 \%)$ & \\
\hline Female & $102(38.9 \%)$ & $46(26.6 \%)$ & \\
\hline \multicolumn{3}{|l|}{ ECOG Performance-Status } & 0.753 \\
\hline $0-1$ & $235(89.7 \%)$ & $153(88.4 \%)$ & \\
\hline$\geq 2$ & $27(10.3 \%)$ & $20(11.6 \%)$ & \\
\hline \multicolumn{3}{|l|}{ Primary tumor site, no (\%) } & 0.162 \\
\hline $\mathrm{ICC}$ & $120(45.8 \%)$ & $88(50.9 \%)$ & \\
\hline $\mathrm{GB} \mathrm{Ca}$ & $89(34.0 \%)$ & $57(32.9 \%)$ & \\
\hline Extrahepatic BTC & $30(11.5 \%)$ & $22(12.7 \%)$ & \\
\hline $\mathrm{AoV} \mathrm{Ca}$ & $23(8.8 \%)$ & $6(3.5 \%)$ & \\
\hline \multicolumn{3}{|l|}{ CEA (ng/mL), no $(\%)$} & 0.038 \\
\hline $\mathrm{CEA} \leq 5$ & $155(68.0 \%)$ & $86(57.3 \%)$ & \\
\hline $\mathrm{CEA}>5$ & $73(32.0 \%)$ & $64(42.7 \%)$ & \\
\hline \multicolumn{3}{|l|}{ CA $19-9(\mathrm{U} / \mathrm{mL})$, no $(\%)$} & 0.678 \\
\hline CA $19-9 \leq 37$ & $17(6.5 \%)$ & $15(8.7 \%)$ & \\
\hline CA $19-9>37$ & $77(29.4 \%)$ & $47(27.2 \%)$ & \\
\hline \multicolumn{3}{|l|}{ PLR, no $(\%)$} & 0.082 \\
\hline PLR $<121$ & $98(52.4 \%)$ & $51(42.1 \%)$ & \\
\hline PLR $\geq 121$ & $89(47.6 \%)$ & $70(57.9 \%)$ & \\
\hline \multicolumn{3}{|l|}{ Serum CRP (mg/dl), no $(\%)$} & 0.683 \\
\hline$<3.3$ & $88(57.5 \%)$ & $52(60.5 \%)$ & \\
\hline$\geq 3.3$ & $65(42.5 \%)$ & $34(39.5 \%)$ & \\
\hline \multicolumn{3}{|l|}{ Albumin $(\mathrm{g} / \mathrm{dl})$, no $(\%)$} & $<0.001$ \\
\hline$\leq 3.3$ & $40(15.7 \%)$ & $57(33.9 \%)$ & \\
\hline$>3.3$ & $215(84.3 \%)$ & $111(66.1 \%)$ & \\
\hline \multicolumn{3}{|l|}{ Metastatic site, no (\%) } & 0.137 \\
\hline$\leq 2$ & $218(83.2 \%)$ & $134(77.5 \%)$ & \\
\hline$>2$ & $44(16.8 \%)$ & $39(22.5 \%)$ & \\
\hline \multicolumn{3}{|l|}{ Histologic Differentiation } & 0.029 \\
\hline Well Differentiated & $12(9.7 \%)$ & $3(5.0 \%)$ & \\
\hline Moderately Differentiated & $69(55.6 \%)$ & $24(40.0 \%)$ & \\
\hline Poorly Differentiated & $43(34.7 \%)$ & $33(55.0 \%)$ & \\
\hline \multicolumn{3}{|l|}{$1^{\text {st }}$ line Chemotherapy ORR } & 0.214 \\
\hline PR or CR & $46(19.7 \%)$ & $21(14.3 \%)$ & \\
\hline & $187(80.3 \%)$ & $126(85.7 \%)$ & \\
\hline \multirow{2}{*}{$\begin{array}{l}\text { SD or PD } \\
\text { PFS (Months) }\end{array}$} & 4.57 & 3.23 & 0.028 \\
\hline & $95 \%$ CI: $3.91-5.23$ & $95 \%$ CI:2.20-4.27 & \\
\hline \multirow[t]{2}{*}{ OS (Months) } & 9.80 & 6.90 & $<0.001$ \\
\hline & $95 \% \mathrm{CI}: 8.97-10.64$ & $95 \%$ CI:5.90-7.90 & \\
\hline
\end{tabular}

Abbreviations: ICC, intrahepatic cholangiocarcinoma; BTC, biliary tract cancer; GB Ca, gallbladder cancer;AoV Ca, ampulla of Vater cancer; ECOG PS, Eastern Cooperative Oncology Group performance status;CEA, carcinoembryonic antigen; CA 19-9, cancer antigen 19-9; NLR, neutrophil-lymphocyte ratio; PLR, platelet-lymphocyte ratio; CRP, C-reactive protein; ORR, objective response rate; $\mathrm{PR}$, partial response; $\mathrm{CR}$, complete response; $\mathrm{SD}$, stable disease; PD, progression disease; OS, overall survival; PFS, progression- free survival; 95\% CI, 95\% confidence interval

rarely been analyzed. Only a few reports are available about NLR and PLR in patients with BTC who received curative surgery [13].

In the case of advanced BTC, little is known about the significance of NLR and PLR.

In this study, we investigated the prognostic role of NLR, PLR and CRP in patients with advanced BTC who received palliative chemotherapy in terms of response to chemotherapy, progression-free survival (PFS), and overall survival (OS). Additionally, we also co-analyzed the dynamics of NLR and PLR during chemotherapy.

\section{RESULTS}

\section{Patients and treatments}

A total of 450 patients with unresectable BTC were enrolled. Patient characteristics are shown in Table 1. The median age was 61 years (range, 26-84 years). The 
Table 3: Comparison between the high PLR and low PLR groups.

\begin{tabular}{|c|c|c|c|}
\hline & $\begin{array}{l}\text { Low PLR group } \\
\text { PLR }<121(\mathrm{~N}=119)\end{array}$ & $\begin{array}{l}\text { High PLR group } \\
\text { PLR } \geq 121(\mathrm{~N}=316)\end{array}$ & $p$ value \\
\hline \multicolumn{3}{|l|}{ Age- yr } & \multirow[t]{3}{*}{0.50} \\
\hline$<65$ & $75(63.0 \%)$ & $210(66.5 \%)$ & \\
\hline$>65$ & $44(37.0 \%)$ & $106(33.5 \%)$ & \\
\hline \multicolumn{3}{|l|}{ Sex, no $(\%)$} & \multirow[t]{3}{*}{0.055} \\
\hline Male & $87(73.1 \%)$ & $200(63.3 \%)$ & \\
\hline Female & $32(26.9 \%)$ & $116(36.7 \%)$ & \\
\hline \multicolumn{3}{|l|}{ ECOG Performance-Status } & \multirow[t]{3}{*}{0.118} \\
\hline $0-1$ & $111(93.3 \%)$ & $277(87.7 \%)$ & \\
\hline$\geq 2$ & $8(6.7 \%)$ & $39(12.3 \%)$ & \\
\hline \multicolumn{3}{|l|}{ Primary tumor site, no (\%) } & \multirow{5}{*}{0.003} \\
\hline $\mathrm{ICC}$ & $74(62.2 \%)$ & $133(42.1 \%)$ & \\
\hline $\mathrm{GB} \mathrm{Ca}$ & $28(23.5 \%)$ & $118(37.3 \%)$ & \\
\hline Extrahepatic BTC & $11(9.2 \%)$ & $41(13.0 \%)$ & \\
\hline $\mathrm{AoV} \mathrm{Ca}$ & $6(5.0 \%)$ & $24(7.6 \%)$ & \\
\hline \multicolumn{3}{|l|}{ CEA (ng/mL), no $(\%)$} & \multirow[t]{3}{*}{0.075} \\
\hline $\mathrm{CEA} \leq 5$ & $103(86.6 \%)$ & $249(78.8 \%)$ & \\
\hline $\mathrm{CEA}>5$ & $16(13.4 \%)$ & $67(21.2 \%)$ & \\
\hline \multicolumn{3}{|l|}{ CA $19-9(\mathrm{U} / \mathrm{mL})$, no $(\%)$} & \multirow{3}{*}{0.280} \\
\hline CA $19-9 \leq 37$ & $39(34.8 \%)$ & $85(29.2 \%)$ & \\
\hline CA $19-9>37$ & $73(65.2 \%)$ & $206(70.8 \%)$ & \\
\hline \multicolumn{3}{|l|}{ Serum CRP (mg/dl), no (\%) } & \multirow[t]{3}{*}{0.568} \\
\hline$<3.3$ & $39(55.7 \%)$ & $101(59.8 \%)$ & \\
\hline$\geq 3.3$ & $31(44.3 \%)$ & $68(40.2 \%)$ & \\
\hline \multicolumn{3}{|l|}{ Albumin $(\mathrm{g} / \mathrm{dl})$, no (\%) } & \multirow[t]{3}{*}{0.052} \\
\hline$\leq 3.3$ & $19(16.4 \%)$ & $78(25.4 \%)$ & \\
\hline$>3.3$ & $97(83.6 \%)$ & $229(74.6 \%)$ & \\
\hline \multicolumn{3}{|l|}{ Metastatic site, no (\%) } & \multirow[t]{3}{*}{0.075} \\
\hline$<2$ & $103(86.6 \%)$ & $249(78.8 \%)$ & \\
\hline$\geq 2$ & $16(13.4 \%)$ & $67(21.2 \%)$ & \\
\hline \multicolumn{3}{|l|}{ Histologic Differentiation } & \multirow[t]{4}{*}{0.748} \\
\hline Well Differentiated & $3(6.0 \%)$ & $12(9.0 \%)$ & \\
\hline Moderately Differentiated & $27(54.0 \%)$ & $66(49.3 \%)$ & \\
\hline Poorly Differentiated & $20(40.0 \%)$ & $56(41.8 \%)$ & \\
\hline \multicolumn{3}{|l|}{$1^{\text {st }}$ line Chemotherapy ORR } & \multirow[t]{3}{*}{0.178} \\
\hline $\mathrm{PR}$ or $\mathrm{CR}$ & $14(13.1 \%)$ & $53(19.4 \%)$ & \\
\hline SD or PD & $93(86.9 \%)$ & $220(80.6 \%)$ & \\
\hline \multirow[t]{2}{*}{ PFS (Months) } & 4.73 & 4.10 & \multirow[t]{2}{*}{0.091} \\
\hline & $95 \%$ CI:3.43-6.03 & $95 \%$ CI: $3.65-4.55$ & \\
\hline \multirow[t]{2}{*}{ OS (Months) } & 9.90 & 7.83 & \multirow[t]{2}{*}{0.012} \\
\hline & $95 \%$ CI 8.78-11.02 & 95\% CI:7.10-8.57 & \\
\hline
\end{tabular}

Abbreviations: ICC, intrahepatic cholangiocarcinoma; BTC, biliary tract cancer; GB Ca, gallbladder cancer;AoV Ca, ampulla of Vater cancer; ECOG PS, Eastern Cooperative Oncology Group performance status;CEA, carcinoembryonic antigen; CA 19-9, cancer antigen 19-9; PLR, platelet-lymphocyte ratio; CRP, C-reactive protein; ORR, objective response rate; PR, partial response; $\mathrm{CR}$, complete response; $\mathrm{SD}$, stable disease; $\mathrm{PD}$, progression disease; OS, overall survival; PFS, progression- free survival; $95 \%$ CI, 95\% confidence interval

study cohort consisted of $295(65.6 \%)$ males and 155 (34.4\%) females. Malignancies included 214 (47.6\%) intrahepatic cholangiocarcinomas (ICC), 151 (33.6\%) gallbladder cancers (GB Ca), 54 (12.0\%) extrahepatic bile duct cancers (Extrahepatic BTC), and 31 (6.9\%) Ampulla of Vater cancers (AoV Ca). The Eastern Cooperative Oncology Group (ECOG) Performance Status (PS) was either 0 or 1 in 401(89.1\%) patients, and PS was 2 in $49(10.9 \%)$ patients. The laboratory values at the time point of advanced BTC diagnosis were as follows: median CEA level, $2.8 \mathrm{ng} / \mathrm{mL}$ (range, 0.5-5410.0 ng/ $\mathrm{mL})$; cancer antigen 19-9(CA 19-9) value, $134.9 \mathrm{U} /$ $\mathrm{mL}$ (range, 1.0-240,0000 U/mL); median total bilirubin, $0.9 \mathrm{mg} / \mathrm{dL}$ (range, $0.1-11.7, \mathrm{mg} / \mathrm{dL}$ ); and albumin, $3.8 \mathrm{~g} /$ $\mathrm{dL}$ (range, 2.1-5.1 g/dL). The median value of NLR was 3.1 (range, 0.5-61.0), and the median value of PLR was 155.8 (range,48.6-4099.0). In terms of tumor origin, the median NLRs were 3.3, 3.1, 3.1, and 2.6 in ICC, GB Ca, 
Table 4: Univariate \& Multivariate Analysis for Overall Survival. OS (median: 8.57 month 95\% CI:7.90-9.24)

\begin{tabular}{|c|c|c|c|c|c|c|}
\hline \multirow[t]{2}{*}{ Variable } & \multicolumn{3}{|c|}{ Univariate analysis } & \multicolumn{3}{|c|}{ Mutivariate } \\
\hline & OS, months & $95 \%$ CI & $p$ value & HR & $95 \%$ CI & $p^{\text {a value }}$ \\
\hline Age $-y r$ & & & 0.004 & & & 0.046 \\
\hline$\leq 65$ & 9.13 & $8.22-10.05$ & & 1 & & \\
\hline$>65$ & 7.67 & $6.17-9.17$ & & 1.317 & $1.005-1.726$ & \\
\hline Sex & & & 0.709 & & & \\
\hline Male & 8.57 & $7.64-9.49$ & & & & \\
\hline Female & 8.63 & $7.74-9.53$ & & & & \\
\hline Primary Tumor Origin & & & 0.244 & & & \\
\hline ICC & 7.80 & $6.74-8.86$ & & & & \\
\hline GB Ca & 9.20 & $8.00-10.40$ & & & & \\
\hline Extrahepatic BTC & 8.57 & 7.39-9.75 & & & & \\
\hline $\mathrm{AoV} \mathrm{Ca}$ & 11.47 & $7.08-15.85$ & & & & \\
\hline ECOG Performance & & & $<0.001$ & & & 0.012 \\
\hline $0-1$ & 8.77 & $8.03-9.51$ & & 1 & & \\
\hline$\geq 2$ & 5.33 & $3.36-7.31$ & & 1.766 & $1.133-2.753$ & \\
\hline CEA (ng/ml) & & & $<0.001$ & & & 0.001 \\
\hline$\leq 5$ & 9.50 & $8.50-10.50$ & & 1 & & \\
\hline$>5$ & 7.23 & $6.19-8.28$ & & 1.679 & $1.232-2.288$ & \\
\hline CA $19-9(\mathrm{U} / \mathrm{ml})$ & & & 0.139 & & & \\
\hline$\leq 37$ & 8.90 & $7.12-10.68$ & & & & \\
\hline$>37$ & 8.63 & $7.86-9.41$ & & & & \\
\hline Albumin (g/dl) & & & 0.022 & & & 0.185 \\
\hline$\leq 3.3$ & 6.90 & $5.73-8.07$ & & 1 & & \\
\hline$>3.3$ & 8.9 & $8.13-9.67$ & & 0.821 & 0.613-1.099 & \\
\hline Serum CRP (mg/dl) & & & 0.162 & & & \\
\hline$<3.3$ & 9.50 & $7.95-11.05$ & & & & \\
\hline$\geq 3.3$ & 7.60 & $6.67-8.54$ & & & & \\
\hline NLR & & & $<0.001$ & & & 0.002 \\
\hline NLR $<3.8$ & 9.80 & $8.97-10.64$ & & 1 & & \\
\hline NLR $\geq 3.8$ & 6.90 & $5.90-7.90$ & & 1.511 & $1.159-1.971$ & \\
\hline PLR & & & 0.012 & & & 0.041 \\
\hline PLR $<121$ & 9.90 & $8.78-11.02$ & & 1 & & \\
\hline$P L R \geq 121$ & 7.83 & $7.10-8.57$ & & 1.352 & $1.013-1.803$ & \\
\hline Change in NLR & & & 0.287 & & & \\
\hline Maintained & 9.93 & $8.89-10.10$ & & & & \\
\hline Increased & 7.67 & $6.08-9.25$ & & & & \\
\hline Change in PLR & & & 0.002 & & & $<0.001$ \\
\hline Maintained & 9.93 & $8.49-11.38$ & & 1 & & \\
\hline Increased & 7.77 & $6.16-9.37$ & & 1.721 & $1.308-2.264$ & \\
\hline
\end{tabular}

Abbreviations: ICC, intrahepatic cholangiocarcinoma; BTC, biliary tract cancer; GB Ca, gallbladder cancer;AoV Ca, ampulla of Vater cancer; ECOG PS, Eastern Cooperative Oncology Group performance status;CEA, carcinoembryonic antigen; CA 19-9, cancer antigen 19-9; NLR, neutrophil-lymphocyte ratio; PLR, platelet-lymphocyte ratio; CRP, C-reactive protein; OS, overall survival;; 95\% CI, 95\% confidence interval $\dagger$ Change in NLR,PLR : maintained $\geq-10 \%$, decreased $<-10 \%$

Extrahepatic BTC, and AoV Ca, respectively. The median PLRs were 139.6, 166.4, 184.3, and 164.6 in ICC, GB $\mathrm{Ca}$, Extrahapatic BTC, and AoV Ca, respectively (Figure
1). 204 patients $(45.3 \%)$ received gemcitabine-based chemotherapy as a first-line treatment, and 232 patients $(51.6 \%)$ received fluoropyrimidine-based chemotherapy 
Table 5: Univariate \& Multivariate Analysis for PFS. PFS (median: 4.20 month 95\% CI:3.75-4.65)

\begin{tabular}{|c|c|c|c|c|c|c|}
\hline \multirow[t]{2}{*}{ Variable } & \multicolumn{3}{|c|}{ Univariate analysis } & \multicolumn{3}{|c|}{ Mutivariate } \\
\hline & PFS, months & $95 \% \mathrm{CI}$ & $p$ value & HR & $95 \% \mathrm{CI}$ & $p^{a}$ value \\
\hline Age - yr & & & 0.504 & & & \\
\hline$\leq 65$ & 4.20 & $3.59-4.82$ & & & & \\
\hline$>65$ & 4.23 & $3.49-4.98$ & & & & \\
\hline Sex & & & 0.768 & & & \\
\hline Male & 4.20 & $3.72-4.58$ & & & & \\
\hline Female & 4.27 & $3.34-5.19$ & & & & \\
\hline Primary Tumor Origin & & & 0.398 & & & \\
\hline $\mathrm{ICC}$ & 3.70 & $2.66-4.74$ & & & & \\
\hline $\mathrm{GB} \mathrm{Ca}$ & 4.53 & $3.92-5.15$ & & & & \\
\hline Extrahepatic BTC & 4.83 & $3.69-5.98$ & & & & \\
\hline $\mathrm{AoV} \mathrm{Ca}$ & 4.43 & $1.33-7.53$ & & & & \\
\hline ECOG Performance Status & & & 0.056 & & & \\
\hline $0-1$ & 4.27 & $3.81-4.72$ & & & & \\
\hline$\geq 2$ & 1.97 & $1.41-2.53$ & & & & \\
\hline $\mathrm{CEA}(\mathrm{ng} / \mathrm{ml})$ & & & 0.042 & & & $<0.001$ \\
\hline$\leq 5$ & 4.43 & $3.99-4.88$ & & 1 & & \\
\hline$>5$ & 4.13 & $2.85-5.42$ & & 1.782 & $1.302-2.439$ & \\
\hline CA 19-9 (U/ml) & & & 0.013 & & & 0.008 \\
\hline$\leq 37$ & 4.53 & $3.80-5.27$ & & 1 & & \\
\hline$>37$ & 4.27 & $3.78-4.75$ & & 1.432 & $1.100-1.863$ & \\
\hline Albumin (g/dl) & & & 0.019 & & & 0.051 \\
\hline$\leq 3.3$ & 3.60 & $2.31-4.89$ & & 1 & & \\
\hline$>3.3$ & 4.37 & $3.88-4.86$ & & 0.734 & $0.539-1.001$ & \\
\hline CRP & & & 0.824 & & & \\
\hline$<3.3$ & 3.67 & $2.58-4.75$ & & & & \\
\hline$>3.3$ & 3.80 & $2.51-5.10$ & & & & \\
\hline NLR & & & 0.028 & & & 0.027 \\
\hline NLR $<3.8$ & 4.57 & $3.91-5.23$ & & 1 & & \\
\hline $\mathrm{NLR} \geq 3.8$ & 3.23 & $2.20-4.27$ & & 1.354 & $1.035-1.772$ & \\
\hline PLR & & & 0.091 & & & \\
\hline PLR $<121$ & 4.73 & $3.43-6.03$ & & & & \\
\hline PLR $\geq 121$ & 4.10 & $3.65-4.56$ & & & & \\
\hline Change in NLR & & & 0.688 & & & \\
\hline Maintained & 4.20 & $3.73-4.67$ & & & & \\
\hline Increased & 3.80 & $2.71-4.88$ & & & & \\
\hline Change in PLR & & & 0.010 & & & 0.009 \\
\hline Maintained & 4.43 & $3.97-4.90$ & & 1 & & \\
\hline Increased & 3.10 & $2.06-4.15$ & & 1.415 & $1.089-1.839$ & \\
\hline ORR & & & $<0.001$ & & & $<0.001$ \\
\hline PR or CR & 8.17 & $7.24-9.10$ & & 1 & & \\
\hline SD or SD & 3.53 & $2.80-4.27$ & & 2.596 & $1.874-3.596$ & \\
\hline
\end{tabular}

Abbreviations: ICC, intrahepatic cholangiocarcinoma; BTC, biliary tract cancer; GB Ca, gallbladder cancer;AoV Ca, ampulla of Vater cancer; ECOG PS, Eastern Cooperative Oncology Group performance status;CEA, carcinoembryonic antigen; CA 19-9, cancer antigen 19-9; NLR, neutrophil-lymphocyte ratio; PLR, platelet-lymphocyte ratio; CRP, C-reactive protein; ORR , objective response rate; PR, partial response; CR, complete response; SD, stable disease; PD, progression disease; OS, overall survival; PFS, progression- free survival; $95 \%$ CI, 95\% confidence interval

Change in NLR,PLR : maintained $\geq-10 \%$, decreased $<-10 \%$

as first-line treatment.

The area under the receiver operating characteristic (ROC) curve for NLR was 0.628 (95\% CI: 0.573-0.683), and an NLR value of 3.8 was identified as the cut-off value for predicting OS, with a sensitivity of $52.3 \%$ and a specificity of $68.4 \%$. The area under the ROC curve for
PLR was 0.592 (95\% CI: 0.536-0.648), and a PLR value of 121 was identified as the cut-off value for predicting OS, with a sensitivity of $78.4 \%$ and a specificity of $33.2 \%$. Additionally, the area under the ROC curve for CRP was 0.552 (95\% CI: 0.477-0.628), and an CRP value of 3.3 was identified as the cut-off value for predicting OS, with 
Table 6: OS analysis of NLR and PLR with co-analysis of dynamics of NLR and PLR during chemotherapy.

\begin{tabular}{|c|c|c|c|c|c|c|c|}
\hline \multirow[b]{2}{*}{ Group } & \multirow[b]{2}{*}{ Change } & \multicolumn{3}{|c|}{ Univariate analysis } & \multicolumn{3}{|c|}{ Mutivariate } \\
\hline & & $\begin{array}{l}\text { OS, } \\
\text { months }\end{array}$ & $95 \% \mathrm{CI}$ & $p$ value & HR & $95 \% \mathrm{CI}$ & $p^{\text {a }}$ value \\
\hline Low NLR group & Maintained & 11.10 & $9.71-12.50$ & $<0.001$ & 1 & & $<0.001$ \\
\hline $\mathrm{NLR}<3.8$ & Increased & 8.67 & $4.87-12.46$ & & 0.992 & $0.708-1.390$ & 0.962 \\
\hline High NLR group & Maintained & 8.43 & $7.39-9.48$ & & 1.399 & $1.036-1.889$ & 0.028 \\
\hline NLR $\geq 3.8$ & Increased & 4.73 & $2.46-7.01$ & & 3.113 & $1.826-5.306$ & $<0.001$ \\
\hline Low PLR group & Maintained & 11.30 & $9.90-12.70$ & 0.001 & 1 & & 0.002 \\
\hline PLR $<121$ & Increased & 7.13 & $0.67-13.59$ & & 1.471 & $0.750-2.887$ & 0.262 \\
\hline High PLR group & Maintained & 7.70 & $6.03-9.37$ & & 1.363 & $0.983-1.891$ & 0.064 \\
\hline PLR $\geq 121$ & Increased & 7.76 & $6.26-9.28$ & & 1.786 & $1.321-2.413$ & $<0.001$ \\
\hline
\end{tabular}

Abbreviations: NLR, neutrophil-lymphocyte ratio; PLR, platelet-lymphocyte ratio

$\dagger$ Change in NLR,PLR : maintained $\geq-10 \%$, decreased $<-10 \%$

$a P$ values were calculated using the Cox-proportional hazard model, age, PS,CEA,albumin,

$49.1 \%$ sensitivity and $50.9 \%$ specificity. The patients were separated into 2 groups according to the cut-off values for NLR and PLR (low: $<3.8$ or high: $\geq 3.8$; low: $<121$ or high $\geq 121$, respectively). Our study showed a strong correlation for NLR and PLR $(\mathrm{r}=0.516, p<0.001)$ (Figure $2)$.However, there was no correlation between NLR and CRP $(p=0.711)$ (Supplementary Figure S1A). Also, there was no correlation between PLR and CRP ( $p=0.452)$ (Supplementary Figure S1B).

OS for all patients was 8.57 months (95\% CI:7.909.24), and PFS was 4.20 months (95\% CI: 3.75-4.70).

\section{Comparisons between high and low NLR groups}

The patients with elevated CEA, low albumin and histologic poorly differentiation were more frequently found in the high NLR group ( $p=0.038, p<0.001, p=$ 0.029 , respectively) (Table 2 ). In contrast, primary tumor site, the response rate, CRP were not different between high and low NLR groups $(p=0.162, p=0.214, p=$ $0.683)$. Patients with high NLR showed worse PFS and OS compared with patients with low NLR (3.23 months vs 4.57 months, $p=0.028 ; 6.90$ months vs 9.80 months, $p<0.001)$.

\section{Comparisons between high and low PLR groups}

Unlike NLR, there were differences between low PLR group and high PLR group according to primary tumors $(p=0.003)$ (Table 3$)$. Also, our study showed CEA, albumin, histologic differentiation had no differences between high and low PLR groups ( $p=0.075$, $p=0.052, p=0.748$, respectively). Patients with high PLR showed worse PFS and OS compared with patients with low PLR (4.10 months vs 4.73 months, $p=0.091 ; 7.83$ months vs 9.90 months, $p=0.012$ ).

\section{NLR, PLR, CPR and other prognostic factors for OS}

In multivariate analysis, NLR $\geq 3.8$ was a significant prognostic factor for OS (hazard ratio [HR]:1.511, 95\% CI: $1.159-1.971, p=0.002)$. PLR $\geq 121$ was a significant prognostic factor for OS (HR:1.352, 95\% CI: 1.013$1.803, p=0.041$ ) (Table 4, Figure 3). Age $>65$, CEA were independently associated with survival in advanced BTC. However, CRP $\geq 3.3$, increased NLR after chemotherapy had no influence on OS ( $p=0.162, p=0.287)$. Whereas, increased PLR after chemotherapy significantly influenced on OS (HR: 1.721, 95\% CI: 1.308-2.264, $p<0.001$ ).

\section{NLR, PLR, and other prognostic factors for PFS}

In multivariate analysis, a high NLR together with an increased PLR after chemotherapy, CEA and CA 19-9 levels, and response rate were significant prognostic factors for PFS (Table 5, Figure 4).

\section{Analysis of NLR and PLR with co-analysis of dynamics of NLR and PLR during chemotherapy}

Analysis of NLR in patients in whom NLR changes had occurred (low NLR with maintained NLR, low NLR with increased NLR, high NLR with maintained NLR, high NLR with increased NLR) revealed that patients with high NLR with increased NLR had the shortest survival ( $p$ $<0.001$; Table 6). Compared with a median OS of 11.10 months for patients with low NLR with maintained NLR, patients with high NLR with increased NLR had the worst OS (median OS 4.73 months; HR 3.113; $p<0.001$; Table 6 , Figure 5). In addition, compared with a median PFS of 4.57 months for patients with low NLR with maintained NLR, patients with high NLR with increased NLR had the 
A

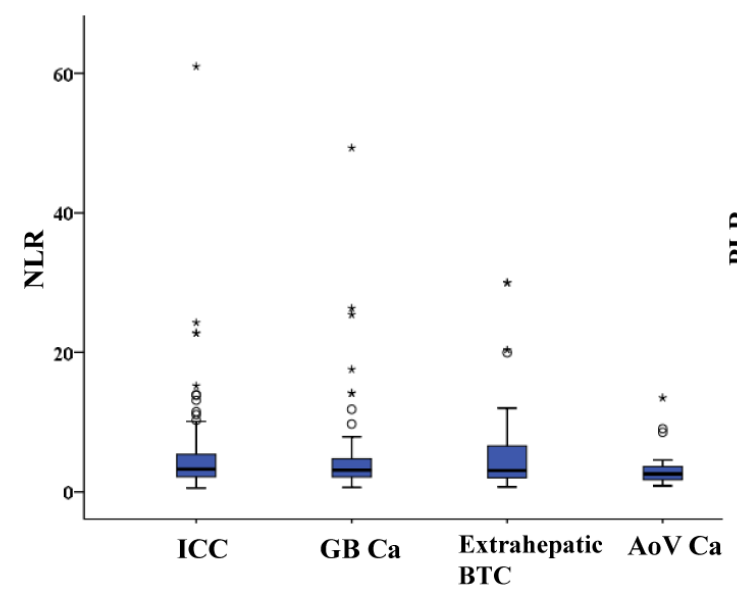

B

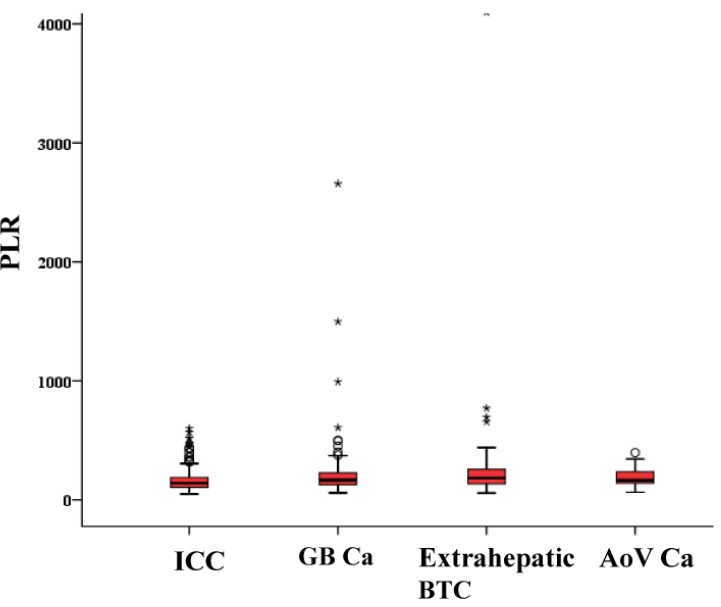

Figure 1: Distribution of NLR,PLR in each subtype of biliary tract cancers. A. Distribution of NLR in each subtype of biliary tract cancers. B. Distribution of PLR in each subtype of biliary tract cancers.

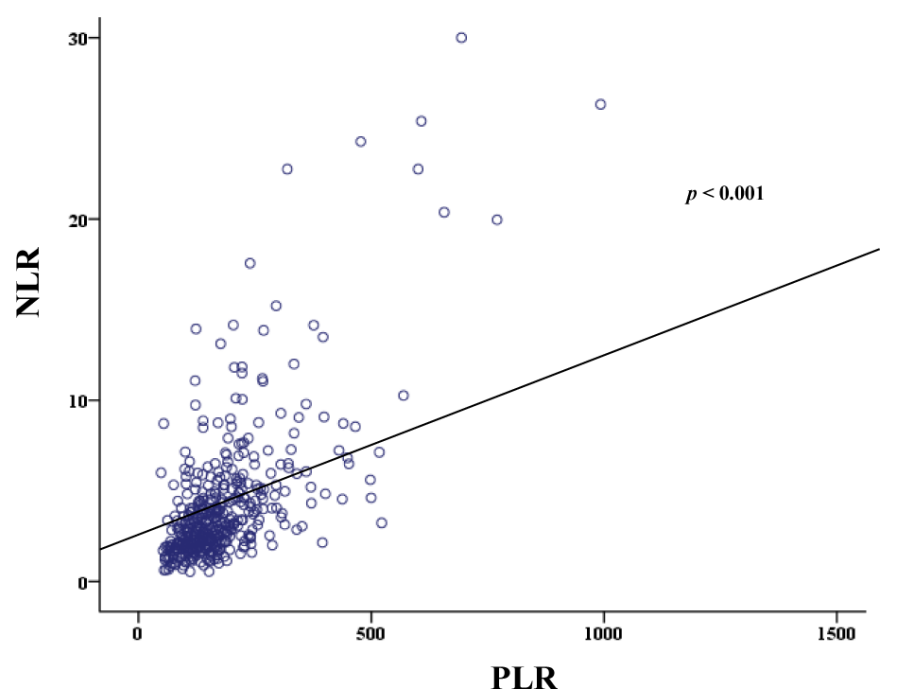

Figure 2: Correlation of NLR,PLR showing distribution of NLR,PLR.

A

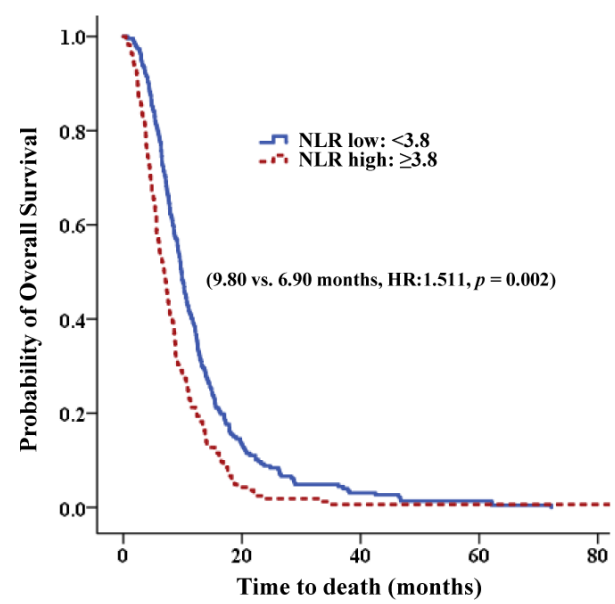

B

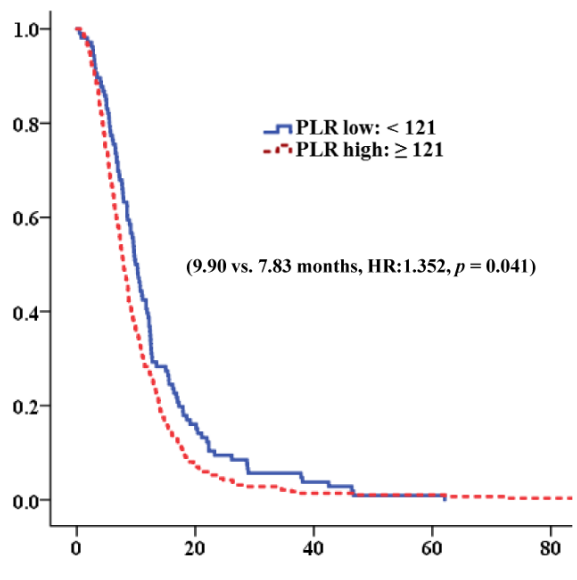

Figure 3: The overall survival according to NLR and PLR. A. The overall survival according to NLR. B. The overall survival according to PLR. 
Table 7: PFS analysis of NLR and PLR with co-analysis of dynamics of NLR and PLR during chemotherapy.

\begin{tabular}{clllllll}
\hline & & \multicolumn{3}{c}{ Univariate analysis } & \multicolumn{3}{c}{ Mutivariate } \\
\cline { 3 - 7 } & & PFS, months & $\mathbf{9 5 \%}$ CI & $\boldsymbol{p}$ value & HR & $\mathbf{9 5 \%}$ CI & $\boldsymbol{p}^{\text {a value }}$ \\
\hline Low NLR group & Maintained & 4.57 & $4.01-5.13$ & $\mathbf{0 . 0 0 1}$ & 1 & & $\mathbf{0 . 0 1 3}$ \\
NLR $<3.8$ & Increased3 & 4.37 & $2.59-6.15$ & & 0.967 & $0.689-1.358$ & 0.848 \\
High NLR group & Maintained4 & 3.70 & $2.49-4.91$ & & 1.426 & $1.037-1.963$ & $\mathbf{0 . 0 2 9}$ \\
NLR $\geq 3.8$ & Increased2 & 1.97 & $1.58-2.36$ & & 2.151 & $1.236-3.744$ & $\mathbf{0 . 0 0 7}$ \\
\hline Low PLR group & Maintained & 4.87 & $4.19-5.55$ & $\mathbf{0 . 0 0 3}$ & 1 & & $\mathbf{0 . 0 0 7}$ \\
PLR $<$ 121 & Increased3 & 4.37 & $0.00-10.66$ & & 0.831 & $0.381-1.812$ & 0.641 \\
High PLR group & Maintained4 & 2.23 & $0.40-4.07$ & & 1.231 & $0.890-1.703$ & 0.209 \\
PLR $\geq 121$ & Increased2 & 3.10 & $2.12-4.08$ & & 1.725 & $1.249-2.381$ & $\mathbf{0 . 0 0 1}$ \\
\hline
\end{tabular}

Abbreviations: NLR, neutrophil-lymphocyte ratio; PLR, platelet-lymphocyte ratio

$\dagger$ Change in NLR,PLR : maintained $\geq-10 \%$, decreased $<-10 \%$

$a P$ values were calculated using the Cox-proportional hazard model, PS, albumin,CEA,CA 19-9, ORR.

worst PFS (median OS 1.97 months; HR 2.151; $p=0.007$; Table 7, Figure 6). Analyses according to PLR showed similar results.

\section{DISCUSSION}

In this study, we found that systemic inflammation represented by NLR and PLR predicts the overall survival of patients with unresectable BTC who are receiving palliative chemotherapy.

Tumor metastasis depends on interactions between tumor cells and the host microenvironment. This involves blood cells, components of the coagulation system, stromal cells, and the extracellular matrix. Cells within the bloodstream include endothelial cells, platelets, lymphocytes, macrophages, mast cells, and fibroblasts [14]. These cells produce cytokines, which are cytotoxic mediators like reactive oxygen species (ROS), tumor necrosis factor-alpha (TNF- $\alpha$ ), and interleukins, leading to tumor progression [15]. High neutrophil counts have long

A

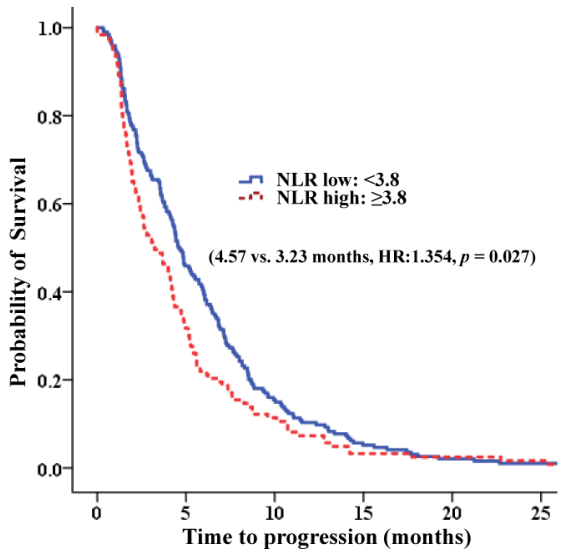

been reported to negatively affect the prognosis of various other cancers [11]. The neutrophils release ROS, which causes point mutations and DNA damage [16]. Also, neutrophils release neutrophil elastase, which is related to tumor cell proliferation, VEGF-related angiogenesis [17]. Through these mechanisms, neutrophils promote proliferation, invasion, and angiogenesis in cancer. Furthermore, $\mathrm{T}$ cells in tumors induce an immune response to the lesion, which includes a high number of cytotoxic $\mathrm{T}$ lymphocytes and increased neoplastic cell apoptosis [18]. Lymphocytopenia suppresses the immune response due to a marked decrease in T4 helper lymphocytes and an increase in T8 suppressor lymphocytes [19].

Platelet count is an additional index of systemic inflammation caused by the tumor. Platelet adhesion and aggregation leads to the formation and release of platelet granules that contain active proteases, growth factors, matrix proteins, and chemokines that enhance tumor progression [20]. Also, platelets release many factors, such as angiopoietin 1, epidermal growth factor, basic fibroblast growth factor, and interleukin-1 $\beta$ and IL- 8 cytokines,

B

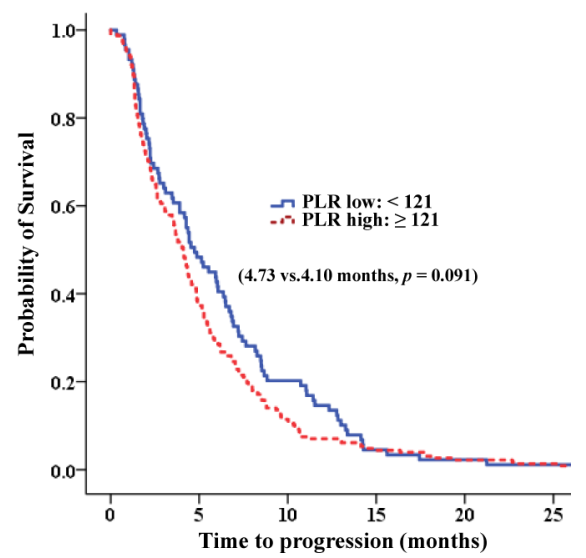

Figure 4: The progression free survival according to NLR and PLR. A. The progression free survival according to NLR. B. The progression free survival according to PLR 
which regulate the angiogenic process [21].

Therefore, NLR and PLR are considered to be the balance between inflammatory status and antitumor status. Patients with elevated NLR and PLR have relative lymphocytopenia, neutrophil leukocytosis, and thrombocytosis, which cause pro-tumor inflammation. Our study showed that NLR is associated with the serum albumin level. Scheede-Bergdahl et al. have reported an association between cancer cachexia and interleukin (IL)-1b levels [22], and Tisdale et al. have also reported that cachexia is associated with cytokines, including tumor necrosis factor-a (TNF-a), IL-1, IL-2, and IL-6, and interferon-r (IFN-r). In light of these findings, NLR could be used as a predictive marker of cancer cachexia. NLR is also significantly associated with CEA, which is an indicator of tumor burden [23]. This suggests that the greater the tumor burden, the greater the NLR, and this is consistent with reports that there is a significant association between higher NLR, larger tumor size, and the extent of micro-vascular invasion in cases of resectable BTC [24]. In addition, NLR is significantly associated with tumor cell differentiation.

In contrast with NLR, PLR varies in BTC according to tumor origin $(p=0.003)$. Compared with other primary tumors, ampulla of Vater cancer $(\mathrm{AoV} \mathrm{Ca})$ has a greater distribution of lower PLR values. Thus, differences in overall survival (OS) according to the primary tumor in BTC might be associated to some extent with PLR. PLR also had a slight association with CEA, CA 19-9, and albumin levels and with tumor cell differentiation.
A

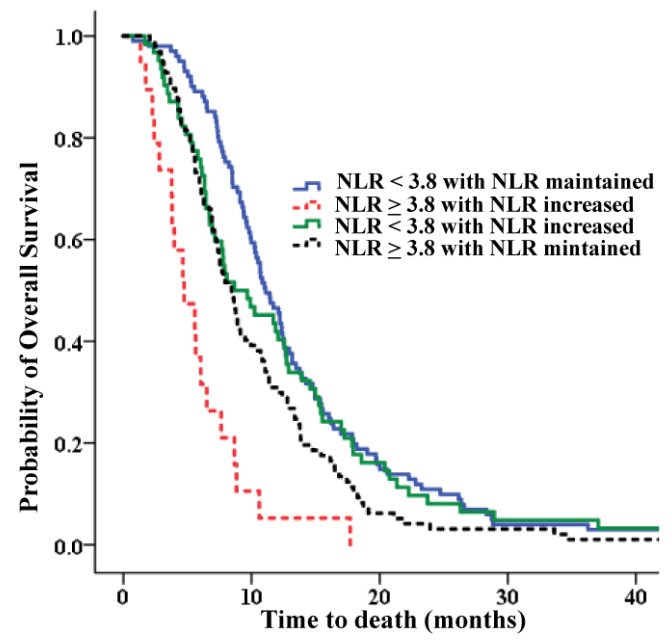

B

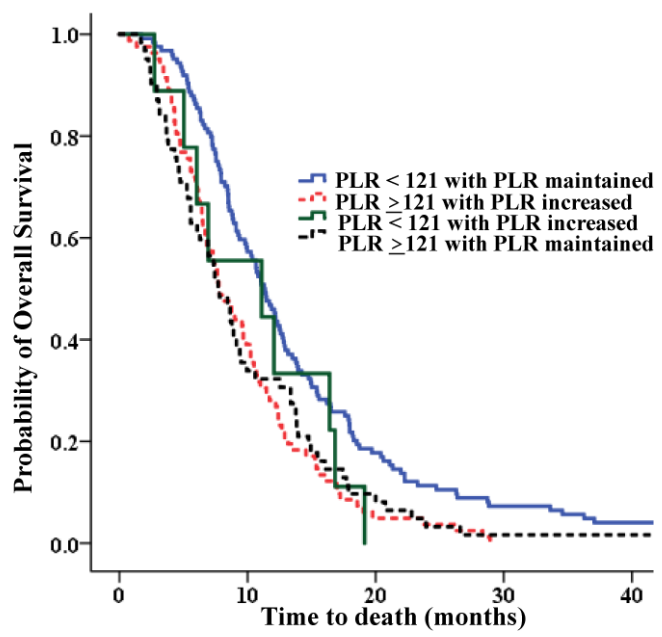

Figure 5: OS analysis of NLR and PLR with co-analysis of dynamics of NLR and PLR. A. OS analysis of NLR with coanalysis of dynamics of NLR. B. OS analysis of PLR with co-analysis of dynamics of PLR.

A

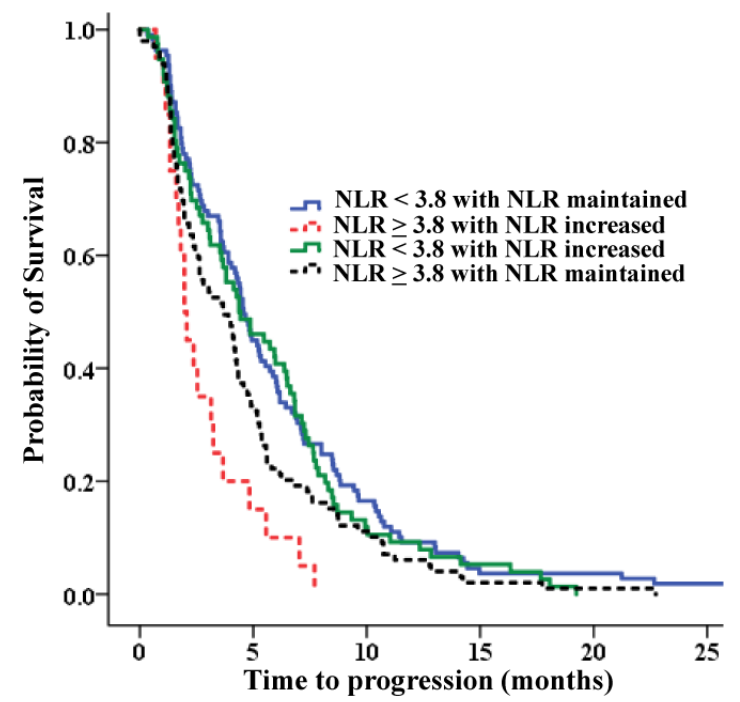

B

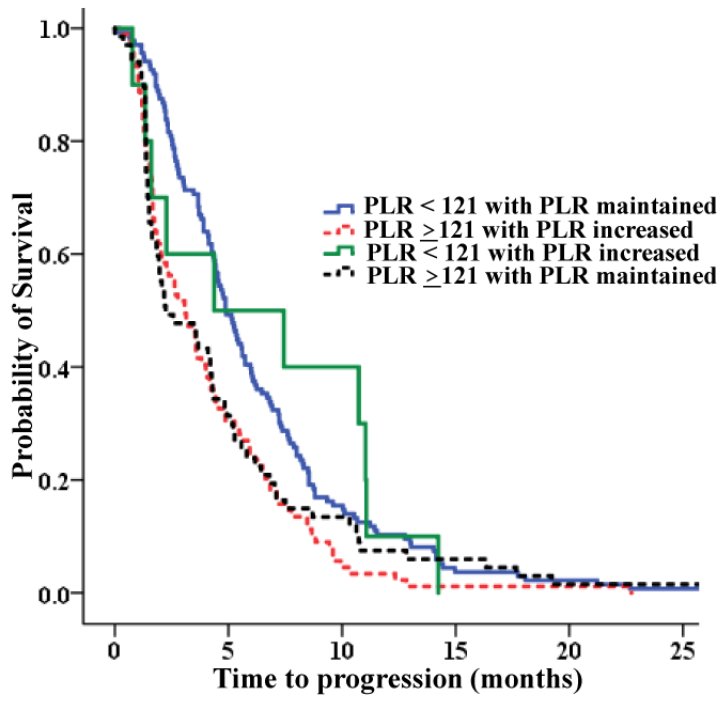

Figure 6: PFS analysis of NLR and PLR with co-analysis of dynamics of NLR and PLR. A. PFS analysis of NLR with coanalysis of dynamics of NLR. B. PFS analysis of PLR with co-analysis of dynamics of PLR. 
Considering the above, when we look at both NLR and PLR, we can more accurately predict the prognosis of BTC.

In a previous report about NLR as a prognostic factor in advanced BTC [25], an association was reported between NLR and OS, but no association was found between NLR and PFS. In our study, an association was found between NLR OS and PFS. Interestingly, in our study, no relationship existed between changes in NLR or PLR. However, when we analyzed subgroups according to NLR and dynamic change in NRL during chemotherapy, our study reveals that high NLR with increased NLR after chemotherapy was associcated with a worse survival outcome (Table 6). There was a 6.4 month OS difference between high NLR with increased NLR group and low NLR with maintained NLR group. The results for PLR are similar. Furthermore, our study reveals that high NLR with increased NLR after chemotherapy is associcated with a worse PFS. So, in light of the above information, considering NLR and PLR along with change might help to more accurately predict heterogeneous prognoses in BTC.

Currently, no consensus exists regarding NLR and PLR cut-off values, which vary across tumor types [12, 26]. In the present study, NLR and PLR cut-off values of 3.8 and 121, respectively, were selected using ROC curves, and the same procedure was used as reported in other studies [26]. McNamara et al. demonstrated a median NLR cut-off value of 3 as a prognostic marker in advanced BTC [25]. Dumitrascu et al. demonstrated a median NLR cut-off value of 3.3 as a prognostic marker in resectable BTC [13].

Recently, there have been several reports showing that circulating tumor cells are associated with prognosis $[23,27]$, and in our previous published reports, the soluble form of programmed death-ligand 1 (PD-L1) in peripheral blood was associated with prognosis in advanced BTC [28]. Further studies to find the association of NLR with circulating stem cells might be worthy.

A limitation of the present study is its retrospective design. Another caveat is that NLR is known to be a nonspecific marker of inflammation. Therefore, the presence of another systemic disease could have influenced the NLR value.

In conclusion, systemic inflammation represented by NLR and PLR predicts the OS of patients with advanced BTC who are receiving palliative chemotherapy. In addition, considering NLR and PLR with dynamic changes of NLR and PLR during chemotherapy might help to predict a more accurate prognosis in advanced BTC.

\section{PATIENTS AND METHODS}

\section{Study population and data collection}

We consecutively enrolled patients with advanced BTC who were diagnosed at the Seoul National University Hospital (Seoul, South Korea) between 2003 and 2013. All patients fulfilled the following criteria: (1) Histologically confirmed biliary tract cancer, (2) Unresectable, metastatic biliary tract cancer, and (3) Had received at least one cycle of palliative chemotherapy, (4) The recurrent biliary tract cancer patients were excluded.

The medical records of each patient were reviewed with respect to laboratory complete blood count (CBC) at the time of diagnosis, sex, age, tumor origin, localization, tumor marker, PFS, and OS.

The NLR was defined as the absolute neutrophil count in peripheral blood divided by the absolute lymphocyte count. The PLR was defined as the absolute platelet count in peripheral blood divided by the absolute lymphocyte count. Changes in NLR, PLR were obtained by subtracting the initial value from the value obtained after progression of chemotherapy.

\section{Statistics}

A receiver operator characteristic (ROC) curve was used to assess the best discriminative cut-off values of NLR, PLR, and CRP while showing an association between NLR, PLR, and OS. The chi-square test was used to compare baseline characteristics of patients between the NLR and PLR groups. Overall survival (OS) was calculated from the first day of diagnosis of advanced BTC to the date of death, and progressionfree survival (PFS) was calculated from the first day of palliative chemotherapy to the date of progression or any cause of death. We analyzed the OS of all patients in this study. The OS and PFS were assessed using KaplanMeier estimates. The log-rank test was used to assess the equality of the survivor function across groups. The Cox proportional hazards model with $95 \%$ confidence interval was used for the multivariate analysis to assess the effect of patient characteristics and other significant prognostic factors such as NLR and PLR. A p value of 0.05 or less was considered statistically significant. All analyses were performed using SPSS software for Windows (version 21; IBM SPSS, Somers, NY).

\section{Ethics}

The study protocol was reviewed and approved by the Institutional Review Board of Seoul National University Hospital (IRB No.H-1306-109-500). All studies were conducted according to guidelines (Declaration of Helsinki) for biomedical research. 


\section{FUNDING SOURCES}

This study was supported by a grant from the National R\&D Program for Cancer Control, Ministry of Health \& Welfare, Republic of Korea to Dr. Do-Youn Oh. (Grant No. 1320090) and SNU Invitation Program for Distinguished Scholar to Dr. Do-Youn Oh.

\section{CONFLICT OF INTEREST}

All authors have no conflict of interest to declare.

\section{REFERENCES}

1. Jemal A, Siegel R, Ward E, Murray T, Xu J, Thun MJ. Cancer statistics, 2007. CA Cancer J Clin. 2007; 57: 43-66.

2. Shin HR, Jung KW, Won YJ, Park JG. 2002 annual report of the Korea Central Cancer Registry: based on registered data from 139 hospitals. Cancer Res Treat. 2004; 36: 103 114.

3. Khan SA, Davidson BR, Goldin RD, Heaton N, Karani J, Pereira SP, Rosenberg WM, Tait P, Taylor-Robinson SD, Thillainayagam AV, Thomas HC, Wasan H. Guidelines for the diagnosis and treatment of cholangiocarcinoma: an update. Gut. 2012; 61: 1657-1669.

4. Alvaro D, Bragazzi MC, Benedetti A, Fabris L, Fava G, Invernizzi P, Marzioni M, Nuzzo G, Strazzabosco M, Stroffolini T. Cholangiocarcinoma in Italy: A national su rvey on clinical characteristics, diagnostic modalities and treatment. Results from the "Cholangiocarcinoma" committee of the Italian Association for the Study of Liver dis ease. Dig Liver Dis. 2011; 43: 60-65.

5. Iwaku A, Kinoshita A, Onoda H, Fushiya N, Nishino H, Matsushima M, Tajiri H. The Glasgow Prognostic Score accurately predicts survival in patients with biliary tract cancer not indicated for surgical resection. Med Oncol. 2014; $31: 787$.

6. Hanahan D, Weinberg RA. Hallmarks of cancer: the next generation. Cell. 2011; 144: 646-674.

7. Patel T. Cholangiocarcinoma. Nat Clin Pract Gastroenterol Hepatol. 2006; 3: 33-42.

8. Lee CH, Chang CJ, Lin YJ, Yeh CN, Chen MF, Hsieh SY. Viral hepatitis-associated intrahepatic cholangiocarcinoma shares common disease processes with hepatocellular carcinoma. Br J Cancer. 2009; 100: 1765-1770.

9. Du Q, Jiang L, Wang X, Wang M, She F, Chen Y. Tumor necrosis factor-alpha promotes the lymphangiogenesis of gallbladder carcinoma through nuclear factor-kappaBmediated upregulation of vascular endothelial growth factor-C. Cancer Sci. 2014; 105: 1261-1271.

10. Wehbe H, Henson R, Meng F, Mize-Berge J, Patel T. Interleukin-6 contributes to growth in cholangiocarcinoma cells by aberrant promoter methylation and gene expression. Cancer Res. 2006; 66: 10517-10524.
11. Bruckner HW, Lavin PT, Plaxe SC, Storch JA, Livstone EM. Absolute granulocyte, lymphocyte, and moncyte counts. Useful determinants of prognosis for patients with metastatic cancer of the stomach. Jama. 1982; 247: 10041006.

12. Gao F, Li X, Geng M, Ye X, Liu H, Liu Y, Wan G, Wang X. Pretreatment neutrophil-lymphocyte ratio: an independent predictor of survival in patients with hepatocellular carcinoma. Medicine (Baltimore). 2015; 94: e639.

13. Dumitrascu T, Chirita D, Ionescu M, Popescu I. Resection for hilar cholangiocarcinoma: analysis of prognostic factors and the impact of systemic inflammation on long-term outcome. J Gastrointest Surg. 2013; 17: 913-924.

14. Joyce JA, Pollard JW. Microenvironmental regulation of metastasis. Nat Rev Cancer. 2009; 9: 239-252.

15. Unal D, Eroglu C, Kurtul N, Oguz A, Tasdemir A. Are neutrophil/lymphocyte and platelet/lymphocyte rates in patients with non-small cell lung cancer associated with treatment response and prognosis? Asian Pac J Cancer Prev. 2013; 14: 5237-5242.

16. Gungor N, Knaapen AM, Munnia A, Peluso M, Haenen GR, Chiu RK, Godschalk RW, van Schooten FJ. Genotoxic effects of neutrophils and hypochlorous acid. Mutagenesis. 2010; 25: 149-154.

17. Scapini P, Morini M, Tecchio C, Minghelli S, Di Carlo E, Tanghetti E, Albini A, Lowell C, Berton G, Noonan DM, Cassatella MA. CXCL1/macrophage inflammatory protein2-induced angiogenesis in vivo is mediated by neutrophilderived vascular endothelial growth factor-A. J Immunol. 2004; 172: 5034-5040.

18. Dolcetti R, Viel A, Doglioni C, Russo A, Guidoboni M, Capozzi E, Vecchiato N, Macri E, Fornasarig M, Boiocchi $\mathrm{M}$. High prevalence of activated intraepithelial cytotoxic $\mathrm{T}$ lymphocytes and increased neoplastic cell apoptosis in colorectal carcinomas with microsatellite instability. Am J Pathol. 1999; 154: 1805-1813.

19. Menges T, Engel J, Welters I, Wagner RM, Little S, Ruwoldt R, Wollbrueck M, Hempelmann G. Changes in blood lymphocyte populations after multiple trauma: association with posttraumatic complications. Crit Care Med. 1999; 27: 733-740.

20. Mueller BM, Reisfeld RA, Edgington TS, Ruf W. Expression of tissue factor by melanoma cells promotes efficient hematogenous metastasis. Proc Natl Acad Sci U S A. 1992; 89: 11832-118836.

21. Coppinger JA, Cagney G, Toomey S, Kislinger T, Belton O, McRedmond JP, Cahill DJ, Emili A, Fitzgerald DJ, Maguire PB. Characterization of the proteins released from activated platelets leads to localization of novel platelet proteins in human atherosclerotic lesions. Blood. 2004; 103: 2096-2104.

22. Scheede-Bergdahl C, Watt HL, Trutschnigg B, Kilgour RD, Haggarty A, Lucar E, Vigano A. Is IL-6 the best proinflammatory biomarker of clinical outcomes of cancer 
cachexia? Clin Nutr. 2012; 31: 85-88.

23. Fang C, Fan C, Wang C, Huang Q, Meng W, Yu Y, Yang L, Peng Z, Hu J, Li Y, Mo X, Zhou Z. CD133+CD54+CD44+ circulating tumor cells as a biomarker of treatment selection and liver metastasis in patients with colorectal cancer. Oncotarget. 2016; 7:77389-77403. doi: 10.18632/ oncotarget. 12675.

24. Gomez D, Morris-Stiff G, Toogood GJ, Lodge JP, Prasad KR. Impact of systemic inflammation on outcome following resection for intrahepatic cholangiocarcinoma. J Surg Oncol. 2008; 97: 513-518.

25. McNamara MG, Templeton AJ, Maganti M, Walter T, Horgan AM, McKeever L, Min T, Amir E, Knox JJ. Neutrophil/lymphocyte ratio as a prognostic factor in biliary tract cancer. Eur J Cancer. 2014; 50: 1581-1599.

26. Jiang L, Jiang S, Situ D, Lin Y, Yang H, Li Y, Long H, Zhou Z. Prognostic value of monocyte and neutrophils to lymphocytes ratio in patients with metastatic soft tissue sarcoma. Oncotarget. 2015; 6: 9542-5095. doi: 10.18632/ oncotarget.3283.

27. Kasimir-Bauer S, Bittner AK, Konig L, Reiter K, Keller T, Kimmig R, Hoffmann O. Does primary neoadjuvant systemic therapy eradicate minimal residual disease? Analysis of disseminated and circulating tumor cells before and after therapy. Breast Cancer Res. 2016; 18: 20.

28. Ha H, Nam AR, Bang JH, Park JE, Kim TY, Lee KH, Han SW, Im SA, Kim TY, Bang YJ, Oh DY. Soluble programmed death-ligand 1 (sPDL1) and neutrophil-tolymphocyte ratio (NLR) predicts survival in advanced biliary tract cancer patients treated with palliative chemotherapy. Oncotarget. 2016; 7:76604-76612. doi: 10.18632/oncotarget.12810. 